\title{
ITERATIVE OPTIMIZATION FOR MAX-MIN SINR IN DENSE SMALL-CELL MULTIUSER MISO SWIPT SYSTEM
}

\author{
Ali Arshad Nasir ${ }^{\dagger}$, Duy Trong Ngo ${ }^{\S}$, Hoang Duong Tuan ${ }^{\ddagger}$ and Salman Durrani ${ }^{\dagger}$ \\ ${ }^{\dagger}$ Research School of Engineering, Australian National University, Canberra, Australia. \\ ${ }^{\S}$ School of Electrical Engineering and Computer Science, The University of Newcastle, Australia. \\ ${ }^{\ddagger}$ Faculty of Engineering and Information Technology, University of Technology Sydney, Australia.
}

\begin{abstract}
Considering a dense small-cell network with simultaneous wireless information and power transfer (SWIPT), this work jointly designs transmit beamformers at the base stations (BSs) and receive power splitting ratios at the users (UEs). Our objectives is to maximize the minimum UE signalto-interference-plus-noise-ratio (SINR) under BS transmit power and UE minimum harvested energy constraints. This problem is highly nonconvex, for which semidefinite programming (SDP) relaxation may even fail to locate a feasible solution. We propose an efficient spectral optimization method by expressing the rank-one constraints as a single reverse convex nonsmooth constraint and incorporating it in the optimization objective. The proposed algorithm practically achieves the theoretical bound given by SDP relaxation with almost similar complexity.
\end{abstract}

Index Terms - Nonsmooth optimization, semidefinite programming, simultaneous wireless information and power transfer, transmit beamforming.

\section{INTRODUCTION}

It is identified that dense small-cell deployment is one of the 'big pillars' to support the much needed $1,000 \times$ increase in data throughput for the $5 \mathrm{G}$ wireless networks [1]. While there is a major concern with the energy consumption of such a dense small-cell deployment, recent advances in wireless power transfer allow the emitted energy in the radio frequency (RF) signals to be harvested and recycled [2]. The scavenged $\mathrm{RF}$ energy is stored in the device battery and later used to power other signal processing and transmitting operations. The simultaneous wireless information and power transfer (SWIPT) from a BS to its UEs is viable in a dense small-cell environment because of the close BS-UE proximity. [3].

Considering SWIPT, [4] proposed a practically realizable receiver structure that separates information decoding and energy harvesting processes by power splitting (PS). In a single-

The work of A. A. Nasir and S. Durrani was supported in part by the Australian Research Councils Discovery Project funding scheme (project number DP14010133) cell MISO network with PS-based receivers and considering the optimization objective of network power minimization, [5-8] jointly designed transmit beamformers at the BS and receive PS ratios at the UEs. Specifically, [5-7] recast the nonconvex sum-power minimization problem as an equivalent semidefinite program (SDP) with rank-one matrix constraints. The common theme in these works is to drop such difficult constraints and solve only the resulting semidefinite relaxation (SDR) problem.

This paper addresses the joint design of transmit beamforming and receive power splitting in a SWIPT-enabled dense small-cell network. Specifically, our optimization objective is to maximize the minimum UE signal-to-interferenceplus-noise-ratio (SINR) under BS transmit power and UE minimum harvested energy constraints. It is easy to check that solving the SDR alone does not return a rank-one solution in general. A relevant question is then how to extract the optimal beamforming vectors from an SDR matrix solution that has rank $r>1$. The randomization method [9] can be employed to find suboptimal vectors from a semidefinite matrix. However, the performance of such a method is inconsistent and it can be poor in many cases [10,11].

In this paper, we propose a new approach that guarantees to find the optimal transmit beamforming vectors and receive power splitting ratios. Basically, we express the rank-one matrix constraints by a single reverse convex constraint, which is then incorporated into the objective as a penalty term. The resulting problem belongs to the class of concave programming [12] with a nonsmooth objective. To solve this problem, we iteratively approximate the objective in the minimization by a convex upper bound, and therefore each iteration only solves one convex SDP. We show that our SDP-based spectral optimization algorithm always converges very quickly, yielding optimal rank-one matrices from which the optimal beamforming vectors are easily recovered.

\section{SYSTEM MODEL AND DESIGN PROBLEM}

We consider the downlink of a dense network consisting of $K$ small cells, where the BS of a cell $k \in \mathcal{K} \triangleq\{1, \ldots, K\}$ 


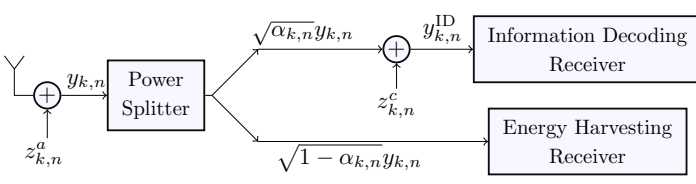

Fig. 1. PS-based receiver structure at UE $(k, n)$.

is equipped with $M>1$ antennas and it serves $N_{k}$ singleantenna UEs within its cell. By BS $k$ and UE $(k, n)$, we mean the BS that serves cell $k$ and the UE $n \in \mathcal{N}_{k} \triangleq\left\{1, \ldots, N_{k}\right\}$ of the same cell, respectively. Given limited radio spectrum availability, we assume universal frequency reuse where all UEs in all cells share the same frequency band. While the spectrum is best utilized in this approach, the signal interference situation among multiple UEs in multiple cells is most severe.

We denote $\mathbf{w}_{\bar{k}, \bar{n}} \in \mathbb{C}^{M \times 1}$ as the beamforming vector by BS $\bar{k} \in \mathcal{K}$ for its UE $(\bar{k}, \bar{n})$ where $\bar{n} \in \mathcal{N}_{\bar{k}} \triangleq\left\{1, \ldots, N_{\bar{k}}\right\}$. Let $\mathbf{h}_{\bar{k}, k, n} \in \mathbb{C}^{M \times 1}$ be the flat fading channel vector between BS $\bar{k}$ and UE $(k, n)$, which includes the effects of large-scale pathloss and small-scale fading. Denote by $x_{\bar{k}, \bar{n}}$ the information signal to be transmitted by $\mathrm{BS} \bar{k}$ to UE $(\bar{k}, \bar{n})$ with $\mathbb{E}\left\{\left|x_{\bar{k}, \bar{n}}\right|^{2}\right\}=1$. The complex baseband signal received by $\mathrm{UE}(k, n)$ is then expressed as

$$
y_{k, n}=\sum_{\bar{k} \in \mathcal{K}} \mathbf{h}_{\bar{k}, k, n}^{H} \sum_{\bar{n} \in \mathcal{N}_{\bar{k}}} \mathbf{w}_{\bar{k}, \bar{n}} x_{\bar{k}, \bar{n}}+z_{k, n}^{a},
$$

where $z_{k, n}^{a} \sim \mathcal{C N}\left(0, \sigma_{a}^{2}\right)$ is the zero-mean circularly complex Gaussian noise with variance $\sigma_{a}^{2}$ at the receiver of $\operatorname{UE}(k, n)$.

As illustrated in Fig. 1, UE $(k, n)$ applies the power splitting (PS) technique to coordinate both information decoding (ID) and energy harvesting (EH). The power splitter divides the received signal $y_{k, n}$ into two parts in the proportion of $\alpha_{k, n}: 1-\alpha_{k, n}$, where $\alpha_{k, n} \in(0,1)$ is termed as the PS ratio for $\operatorname{UE}(k, n)$. The first part $\sqrt{\alpha_{k, n}} y_{k, n}$ forms an input to the ID receiver, where $z_{k, n}^{c} \sim \mathcal{C N}\left(0, \sigma_{c}^{2}\right)$ is the additional noise introduced by the ID receiver circuitry. Therefore, the SINR at the input of the ID receiver of $\mathrm{UE}(k, n)$ is given by (2) at the bottom of the page. The second part $\sqrt{1-\alpha_{k, n}} y_{k, n}$ of the received signal is processed by an EH receiver. With energy harvesting efficiency $\zeta_{k, n} \in(0,1)$, the energy harvested $E_{k, n}$ at $\mathrm{UE}(k, n)$ is given by

$$
E_{k, n} \triangleq \zeta_{k, n}\left(1-\alpha_{k, n}\right)\left(\sum_{\bar{k} \in \mathcal{K}} \sum_{\bar{n} \in \mathcal{N}_{\bar{k}}}\left|\mathbf{h}_{\bar{k}, k, n}^{H} \mathbf{w}_{\bar{k}, \bar{n}}\right|^{2}+\sigma_{a}^{2}\right)
$$

In this paper, we aim to jointly optimize the transmit beamforming vectors $\mathbf{w}_{k, n}$ and the PS ratios $\alpha_{k, n}, \forall k, n$ and consider the following max-min SINR problem.

$$
\begin{aligned}
& \max _{\substack{\mathbf{w}_{k, n} \in \mathbb{C}^{M \times 1} \\
\alpha_{k, n} \in(0,1)}} F(\mathbf{w}, \alpha) \triangleq \min _{k \in \mathcal{K}, n \in \mathcal{N}_{k}} f_{k, n}\left(\mathbf{w}, \alpha_{k, n}\right) \\
& \text { s.t. } \sum_{n \in \mathcal{N}_{k}}\left\|\mathbf{w}_{k, n}\right\|^{2} \leq P_{k}^{\max }, \forall k \in \mathcal{K} \\
& \sum_{k \in \mathcal{K}} \sum_{n \in \mathcal{N}_{k}}\left\|\mathbf{w}_{k, n}\right\|^{2} \leq P^{\max } \\
& E_{k, n}\left(\mathbf{w}, \alpha_{k, n}\right) \geq e_{k, n}^{\min }, \forall k \in \mathcal{K}, n \in \mathcal{N}_{k},
\end{aligned}
$$

where we denote $\mathbf{w} \triangleq\left[\mathbf{w}_{k, n}\right]_{k \in \mathcal{K}, n \in \mathcal{N}_{k}}$ and $\boldsymbol{\alpha} \triangleq\left[\alpha_{k, n}\right]_{k \in \mathcal{K}, n \in \mathcal{N}_{k}}$. Constraint (4b) caps the total transmit power of each BS $k$ at a predefined value $P_{k}^{\max }$. Constraint $(4 \mathrm{c})$ ensures that the total transmit power of the network not exceed the allowable budget $P^{\max }$. Constraint (4d) ensures target harvested energy $e_{k, n}^{\min }$. In summary, (4) is a nonconvex nonsmooth (due to the minimization operator) optimization function subject to nonconvex constraint, i.e., (4d). In the following section, we solve max-min SINR problem (4). Upon defining $\mathbf{W}_{k, n} \triangleq \mathbf{w}_{k, n} \mathbf{w}_{k, n}^{H} \succcurlyeq \mathbf{0}$ and $\mathbf{H}_{k, k, n} \triangleq \mathbf{h}_{k, k, n} \mathbf{h}_{k, k, n}^{H}$ and introducing a new variable $\gamma$, problem (4) is recast as:

$$
\begin{aligned}
\max _{\substack{\mathbf{W}_{k, n} \in \mathbb{C}^{M} \times M \\
\alpha_{k, n} \in(0,1), \gamma}} & \gamma \\
\text { s.t. } & \frac{1}{\gamma} \operatorname{Tr}\left\{\mathbf{H}_{k, k, n} \mathbf{W}_{k, n}\right\}-\sum_{\bar{k} \in \mathcal{K} \backslash\{k\}} \sum_{\bar{n} \in \mathcal{N}_{\bar{k}}} \operatorname{Tr}\left\{\mathbf{H}_{\bar{k}, k, n} \mathbf{W}_{\bar{k}, \bar{n}}\right\} \\
& -\sum_{\bar{n} \in \mathcal{N}_{k} \backslash\{n\}} \operatorname{Tr}\left\{\mathbf{H}_{k, k, n} \mathbf{W}_{k, \bar{n}}\right\} \geq \sigma_{a}^{2}+\frac{\sigma_{c}^{2}}{\alpha_{k, n}}, \forall k \in \mathcal{K}, n \in \mathcal{N}_{k} \\
& \sum_{n \in \mathcal{N}_{k}} \operatorname{Tr}\left\{\mathbf{W}_{k, n}\right\} \leq P_{k}^{\max }, \forall k \in \mathcal{K} \\
& \sum_{k \in \mathcal{K}} \sum_{n \in \mathcal{N}_{k}} \operatorname{Tr}\left\{\mathbf{W}_{k, n}\right\} \leq P^{\max } \\
& \sum_{\bar{k} \in \mathcal{K}} \sum_{\bar{n} \in \mathcal{N}_{\bar{k}}} \operatorname{Tr}\left\{\mathbf{H}_{\bar{k}, k, n} \mathbf{W}_{\bar{k}, \bar{n}}\right\} \geq \frac{e_{k, n}^{\min }}{\zeta_{k, n}\left(1-\alpha_{k, n}\right)}-\sigma_{a}^{2}, \forall k, n \\
& \mathbf{W}_{k, n} \succcurlyeq \mathbf{0}, \forall k \in \mathcal{K}, n \in \mathcal{N}_{k} \\
& \operatorname{rank}\left(\mathbf{W}_{k, n}\right)=1, \forall k \in \mathcal{K}, n \in \mathcal{N}_{k} .
\end{aligned}
$$

Let us also denote $\mathbf{W} \triangleq\left[\mathbf{W}_{k, n}\right]_{k \in \mathcal{K}, n \in \mathcal{N}_{k}}$. Then, constraints (5c)-(5f) are convex in $(\mathbf{W}, \boldsymbol{\alpha}, \gamma)$ since $\frac{1}{1-\alpha_{k, n}}$ is convex on $\alpha_{k, n} \in(0,1)$. In solving (5), it remains to deal with the nonconvex constraints $(5 \mathrm{~b})$ and $(5 \mathrm{~g})$. For a fixed $\gamma,(5 \mathrm{~b})$ is convex in $\left(\mathbf{W}, \alpha_{k, n}\right)$ because $\frac{1}{\alpha_{k, n}}$ is convex on $\alpha_{k, n} \in(0,1)$. By fixing $\gamma$ and further ignoring the difficult rank-one constraint (5g), (5) becomes a feasibility (convex) semidefinite

$$
\operatorname{SINR}_{k, n}=f_{k, n}\left(\mathbf{w}, \alpha_{k, n}\right) \triangleq \underbrace{\frac{\alpha_{k, n}\left|\mathbf{h}_{k, k, n}^{H} \mathbf{w}_{k, n}\right|^{2}}{\alpha_{k, n} \sum_{\bar{n} \in \mathcal{N}_{k} \backslash\{n\}}\left|\mathbf{h}_{k, k, n}^{H} \mathbf{w}_{k, \bar{n}}\right|^{2}}}_{\text {intracell interference }}+\underbrace{\alpha_{k, n} \sum_{\bar{k} \in \mathcal{K} \backslash\{k\}} \sum_{\bar{n} \in \mathcal{N}_{\bar{k}}}\left|\mathbf{h}_{\bar{k}, k, n}^{H} \mathbf{w}_{\bar{k}, \bar{n}}\right|^{2}+\alpha_{k, n} \sigma_{a}^{2}+\sigma_{c}^{2}}_{\text {intercell interference }} .
$$


relaxation (SDR) (5b)-(5f) which can be efficiently solved. Because (5b) is the only constraint that involves $\gamma$ and it is monotonic in $\gamma$, the optimal value of $\gamma$ can be found via a bisection search.

If one is lucky enough to have $\operatorname{rank}\left(\mathbf{W}_{k, n}^{\star}\right)=1, \forall k \in$ $\mathcal{K}, n \in \mathcal{N}_{k}$, the rank-one constraint (5g) is automatically satisfied. The optimal beamforming vector $\mathbf{w}_{k, n}^{\star}$ of (4) can then be recovered from the eigenvalue decomposition of $\mathbf{W}_{k, n}^{\star}$, and its optimal PS ratio is simply $\alpha_{k, n}^{\star}$. However, the trouble arises when $\operatorname{rank}\left(\mathbf{W}_{k, n}^{\star}\right)>1$. Unfortunately this is often true and in such cases, $\gamma^{\star}$ serves only as an upper bound to the optimum of (5) and hence of (4). Our simulation results in Sec. 4 further confirm that the solution of $(5 a)-(5 f)$ in a three-cell network has $\operatorname{rank}\left(\mathbf{W}_{k, n}^{\star}\right)>1$ for some $(k, n)$ in more than $38 \%$ of the time. Clearly, solving the feasibility SDR (5a)-(5f) alone is not adequate to recover the optimal solution $\left(\mathbf{w}_{k, n}^{\star}, \alpha_{k, n}^{\star}\right)$ of (4). In the case of $\operatorname{rank}\left(\mathbf{W}_{k, n}^{\star}\right)>1$, the current approach in the literature is to employ randomization to extract the beamforming vector $\mathbf{w}_{k, n}$ from $\mathbf{W}_{k, n}^{\star}$ [9], however, the generated solutions are not guaranteed to be even close to the actual optimum of problem (5). Motivated by the shortcomings of existing solutions, in what follows we propose an algorithm to solve the challenging problem (4).

\section{PROPOSED SOLUTION}

We follow the approach of [10] to find the optimal rank-one solutions of (4). For any matrix $\mathbf{W}_{k, n} \succcurlyeq \mathbf{0}$, it is true that

$$
\operatorname{Tr}\left\{\mathbf{W}_{k, n}\right\}-\lambda_{\max }\left\{\mathbf{W}_{k, n}\right\} \geq 0,
$$

where $\lambda_{\max }\{\cdot\}$ stands for the maximum eigenvalue of a matrix. Therefore, the rank-one constraints $(5 \mathrm{~g})$ can be expressed by the following single constraint $[10,13]$ :

$$
\sum_{k \in \mathcal{K}} \sum_{n \in \mathcal{H}_{k}}\left[\operatorname{Tr}\left\{\mathbf{W}_{k, n}\right\}-\lambda_{\max }\left\{\mathbf{W}_{k, n}\right\}\right] \leq 0, \forall k, n .
$$

This is because if (7) holds then $\operatorname{Tr}\left\{\mathbf{W}_{k, n}\right\}-\lambda_{\max }\left\{\mathbf{W}_{k, n}\right\}=$ 0 for all $k \in \mathcal{K}$ and $n \in \mathcal{N}_{k}$, which means that each $\mathbf{W}_{k, n}$ has exactly one nonzero eigenvalue. Note that (7) is a reverse convex constraint because the function $\lambda_{\max }\{\cdot\}$ is convex on the set of Hermitian matrices [14, p.147]. For a given $\gamma$, the feasibility problem (5) is thus a convex program with an additional reverse convex constraint, which is an important class of nonconvex optimization [12].

For sufficiently small values of $\operatorname{Tr}\left\{\mathbf{W}_{k, n}\right\}-\lambda_{\max }\left\{\mathbf{W}_{k, n}\right\}$, we have that $\mathbf{W}_{k, n} \approx \lambda_{\max }\left\{\mathbf{W}_{k, n}\right\} \mathbf{w}_{k, n}^{\max }\left(\mathbf{w}_{k, n}^{\max }\right)^{H}$, where $\mathbf{w}_{k, n}^{\max }$ is the unit-norm eigenvector corresponding to the maximum eigenvalue $\lambda_{\max }\left\{\mathbf{W}_{k, n}\right\}$. In this case, the optimal beamforming of problem (4) is then simply:

$$
\mathbf{w}_{k, n}^{\star}=\left(\lambda_{\max }\left\{\mathbf{W}_{k, n}\right\}\right)^{1 / 2} \mathbf{w}_{k, n}^{\max } .
$$

Our aim is thus to make $\sum_{k \in \mathcal{K}} \sum_{n \in \mathcal{N}_{k}}\left[\operatorname{Tr}\left\{\mathbf{W}_{k, n}\right\}-\lambda_{\max }\left\{\mathbf{W}_{k, n}\right\}\right]$ s a convex majorant minimization of the nonconvex program as small as possible. To this end, we incorporate the reverse

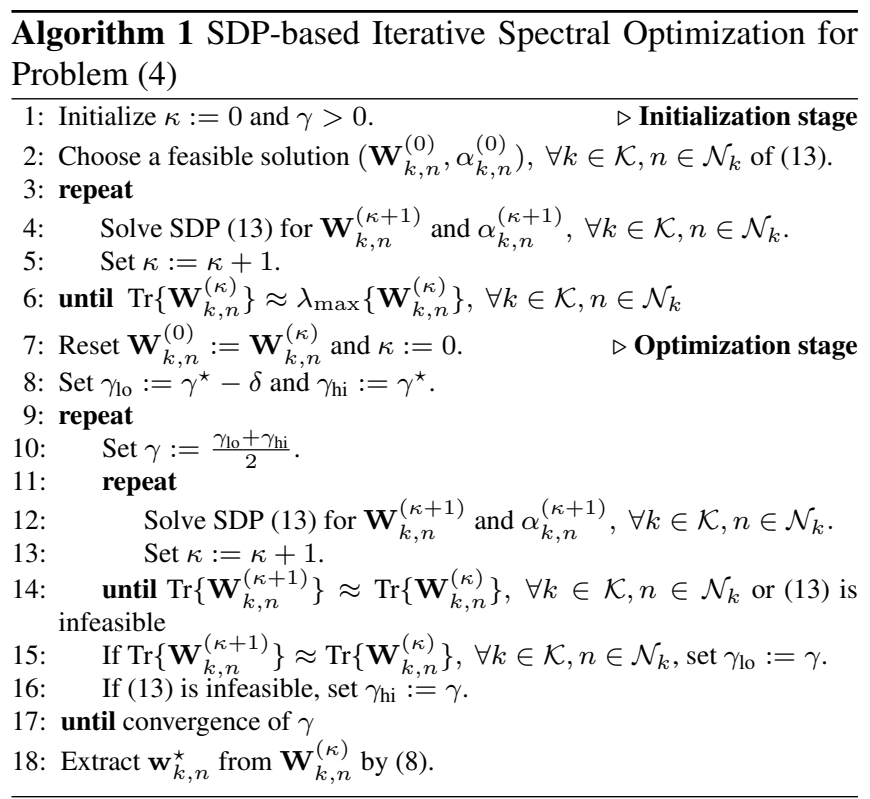

convex constraint (7) into the objective as a penalty function. Thus, for a given $\gamma$, problem (5) is reformulated as:

$$
\begin{array}{ll}
\min _{\substack{\mathbf{W}_{k, n} \in \mathbb{C}^{M \times M} \\
\alpha_{k, n} \in(0,1)}} \tilde{F}(\mathbf{W}) \triangleq \sum_{k \in \mathcal{K}} \sum_{n \in \mathcal{N}_{k}} \operatorname{Tr}\left\{\mathbf{W}_{k, n}\right\}-\lambda_{\max }\left\{\mathbf{W}_{k, n}\right\} \\
& \text { s.t. } \quad(5 \mathrm{~b})-(5 \mathrm{f}) .
\end{array}
$$

Problem (9) involves the minimization of a concave function over a convex set. Furthermore, because $\lambda_{\max }\left\{\mathbf{W}_{k, n}\right\}$ is nonsmooth (i.e., non-differentiable), so is the objective function gramming with a nonsmooth objective [12]. Since the subgradient of $\lambda_{\max }\left\{\mathbf{W}_{k, n}\right\}$ is $\mathbf{w}_{k, n}^{\max }\left(\mathbf{w}_{k, n}^{\max }\right)^{H}[13,15]$, we have

$$
\begin{aligned}
\lambda_{\max }\left\{\mathbf{X}_{k, n}\right\} & \geq \lambda_{\max }\left\{\mathbf{W}_{k, n}\right\} \\
& +\left(\mathbf{w}_{k, n}^{\max }\right)^{H}\left(\mathbf{X}_{k, n}-\mathbf{W}_{k, n}\right) \mathbf{w}_{k, n}^{\max }, \forall k, n
\end{aligned}
$$

for any $\mathbf{X}_{k, n} \succcurlyeq \mathbf{0}$. As such, given some feasible $\mathbf{W}_{k, n}^{(\kappa)}$ of (9) at iteration $\kappa$ with the corresponding maximum eigenvalue $\lambda_{\max }\left\{\mathbf{w}_{k, n}^{\max ,(\kappa)}\right\}$ and unit-norm eigenvector $\mathbf{w}_{k, n}^{\max ,(\kappa)}$ and

$$
\begin{aligned}
\tilde{F}^{(\kappa)}(\mathbf{W}) & \triangleq \sum_{k \in \mathcal{K}} \sum_{n \in \mathcal{N}_{k}} \operatorname{Tr}\left\{\mathbf{W}_{k, n}\right\}-\lambda_{\max }\left\{\mathbf{W}_{k, n}^{(\kappa)}\right\} \\
& -\left(\mathbf{W}_{k, n}^{\max ,(\kappa)}\right)^{H}\left(\mathbf{W}_{k, n}-\mathbf{W}_{k, n}^{(\kappa)}\right) \mathbf{w}_{k, n}^{\max ,(\kappa)} \\
& \geq F(\mathbf{W}), \forall \mathbf{W}
\end{aligned}
$$

the following SDP

$$
\min _{\substack{\mathbf{w}_{k, n} \in \mathbb{C}^{M \times M} \\ \alpha_{k, n} \in(0,1)}} \tilde{F}^{(\kappa)}(\mathbf{W}) \quad \text { s.t. } \quad(5 \mathrm{~b})-(5 \mathrm{f}) .
$$
(9). Problem (9) therefore belongs to the class of concave pro- 


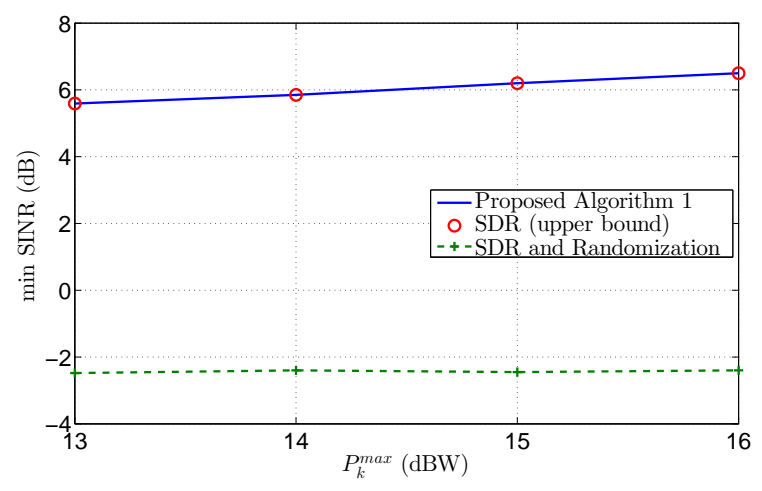

Fig. 2. Maximized minimum UE SINR versus $P_{k}^{\max }$.

is a better solution to (9) than $\left(\mathbf{W}^{(\kappa)}, \boldsymbol{\alpha}^{(\kappa)}\right)$ as [16]

$\tilde{F}\left(\mathbf{W}^{(\kappa+1)}\right) \leq \tilde{F}^{(\kappa)}\left(\mathbf{W}^{(\kappa+1)}\right)<\tilde{F}^{(\kappa)}\left(\mathbf{W}^{(\kappa)}\right)=\tilde{F}\left(\mathbf{W}^{(\kappa)}\right)$

as far as $\left(\mathbf{W}_{k, n}^{(\kappa+1)}, \alpha_{k, n}^{(\kappa+1)}\right) \neq\left(\mathbf{W}_{k, n}^{(\kappa)}, \alpha_{k, n}^{(\kappa)}\right)$ for some $(k, n)$. Program (12) can be further simplified to:

$\min _{\substack{\mathbf{w}_{k, n} \in \mathbb{C}^{M} \times M \\ \alpha_{k, n} \in(0,1)}} \sum_{k \in \mathcal{K}} \sum_{n \in \mathcal{N}_{k}} \operatorname{Tr}\left\{\mathbf{W}_{k, n}\right\}-\left(\mathbf{w}_{k, n}^{\max ,(\kappa)}\right)^{H} \mathbf{W}_{k, n} \mathbf{w}_{k, n}^{\max ,(\kappa)}$

$$
\text { s.t. } \quad(5 b)-(5 f) \text {. }
$$

With (13), we then propose to use a bisection search in an outer loop to find the optimal value of $\gamma$.

In Algorithm 1, we propose the proposed SDP-based iterative algorithm to solve the max-min SINR problem (4). Given an initial feasible point of the feasibility set, we only need to solve one SDP (13) in each iteration, the solution of which will again be used in the next iteration to find an improved solution. The Initialization stage of Algorithm 1 is to find a good feasible solution $\left(\mathbf{W}_{k, n}^{(0)}, \alpha_{k, n}^{(0)}\right)$. In Step 2, $\mathbf{W}_{k, n}^{(0)}$ and $\alpha_{k, n}^{(0)}$ are found by solving the feasibility SDP (5b)(5f). Steps $3-6$ give a feasible solution with $\operatorname{rank}\left(\mathbf{W}_{k, n}\right)=$ $1, \forall k \in \mathcal{K}, n \in \mathcal{N}_{k}$ [10]. In the Optimization stage, the inner loop optimizes $\mathbf{W}_{k, n}, \alpha_{k, n}, \forall k \in \mathcal{K}, n \in \mathcal{N}_{k}$ for a given value of $\gamma$ by solving exactly one SDP (13) in each iteration. The outer loop optimizes $\gamma$ via a simple bisection search. The upper and lower limits for the bisection search are set as $\gamma_{\mathrm{hi}}=\gamma^{\star}$ and $\gamma_{\mathrm{lo}}=\gamma^{\star}-\delta$, where $\delta>0$ and $\gamma^{\star}$ is the optimal upper bound obtained by solving (5b)-(5f) ${ }^{1}$. After $\gamma$ converges, the optimal beamforming vector $\mathbf{w}_{k, n}^{\star}$ of problem (4) is recovered from the optimal matrix $\mathbf{W}_{k, n}^{\text {opt }} \triangleq \mathbf{W}_{k, n}^{(\kappa)}$ of Algorithm 1 according to (8).

\section{NUMERICAL RESULTS}

In our numerical examples, we consider $K=3$ cells, $N_{k}=4$ UEs per cell, and $M=4$ antennas at each BS. We set the

\footnotetext{
${ }^{1}$ We suggest to choose $\delta$ to be small because our simulation results show that the optimal $\gamma$ given by Algorithm 1 is always very close to $\gamma^{\star}$.
}

cell radius as $40 \mathrm{~m}$ and the BS-to-UE distance as $20 \mathrm{~m}$ to simulate a small-cell network deployment, which enables practical SWIPT $[3,17]$. We set the path loss exponent as $\beta=4$. For small-scale fading, we consider the Rician fading channel with Rician factor $=10 \mathrm{~dB}$. For simplicity and without loss of generality, we assume that $\gamma_{k, n}^{\min }=\underline{\gamma}, \zeta_{k, n}=\zeta, e_{k, n}^{\min }=\underline{e}$, $\forall k, n$. We set $\zeta=0.5, \underline{e}=-20 \mathrm{dBm}, \sigma_{a}^{2}=-90 \mathrm{dBm}$ and $\sigma_{c}^{2}=-90 \mathrm{dBm}$. After fine tuning, we set $\delta=1$. We set $P^{\max }=22 \mathrm{dBW}$. Unless specified otherwise, we assume $P_{k}^{\max }=16 \mathrm{dBW}, \forall k \in \mathcal{K}$.

We observe that solving an SDR fails to deliver a rankone solution in $38.3 \%$ of the time on average while the proposed Algorithm 1 always deliver a rank-one solution. In our simulations, we establish that a matrix is only of rank one if the magnitude of its second largest eigenvalue is less than $\rho=1 / 200$ of that of its largest eigenvalue. Since this criterion is much more relaxed than conventionally where $\rho$ is much smaller, it ensures that a rank-one matrix is not mistaken. Fig. 2 considers the case that $\operatorname{rank}\left(\mathbf{W}_{k, n}^{\star}\right)>1, \forall k, n$. It is clear that the performance of the SDR-randomization method is quite far from the optimal upper bound given by SDR. In contrast, our proposed Algorithm 1 achieves this bound, suggesting that a globally optimal solution has been found. Note that the SDR approach fails to guarantee a rankone $\mathbf{W}_{k, n}^{\star}$ solution. If we consider the other case where SDR delivers $\operatorname{rank}\left(\mathbf{W}_{k, n}^{\star}\right)=1, \forall k, n$, there is no need of applying randomization, however the proposed Algorithm 1 still achieves the optimal upper bound given by SDR.

Table 1. Computational complexity analysis in Problem (4)

\begin{tabular}{|c||c|c|c|c|c|}
\hline Alg. & out-iter & in-iter & scal var & lin cons & SD cons \\
\hline \hline SDR & 16 & 1 & 132 & 28 & 36 \\
\hline Alg. 1 & 7.3 & 2 & 132 & 28 & 36 \\
\hline
\end{tabular}

Table 1 shows the average number of iterations for inner and outer loops and the number of scalar variables ('scal var'), linear constraints ('lin cons'), and semidefinite constraints ('SD cons') of the concerned algorithms. It can be observed that the complexity of the proposed Algorithm 1 is very close to that of the SDR method. In Table 1, we do include the computational complexity of SDR-randomization method since its performance is far from optimal.

\section{CONCLUSION}

In this paper, we addressed the joint design of transmit beamforming and receive PS in a SWIPT-enabled dense smallcell network. The conventional approach is not applicable in this case due to the inevitable rank-one matrix constraints. We have therefore, proposed a new iterative optimization approach that offers maximized minimum SINR among all UEs. The optimal solution provided by our SDP-based spectral optimization achieves the theoretical bound. Numerical examples have confirmed the efficiency of our novel method. 


\section{REFERENCES}

[1] J.G. Andrews, S. Buzzi, Wan Choi, S.V. Hanly, A. Lozano, A.C.K. Soong, and J.C. Zhang, "What will 5G be?," vol. 32, no. 6, pp. 1065-1082, Jun. 2014.

[2] X. Lu, P. Wang, D. Niyato, D. I. Kim, and Z. Han, "Wireless networks with RF energy harvesting: A contemporary survey," IEEE Commun. Surveys Tuts., 2015 (to appear).

[3] Jian Huang, Hong Zhang, Wei Xu, and Hua Zhang, "Grouping based inter-cell interference coordination in LTE-A dense small-cell networks," in Proc. IEEE MAPE, Oct. 2013, pp. 78-83.

[4] X. Zhou, R. Zhang, and C. K. Ho, "Wireless information and power transfer: Architecture design and rate-energy tradeoff," IEEE Trans. Commun., vol. 61, no. 11, pp. 4757-4767, Nov. 2013.

[5] Zhengyu Zhu, Zhongyong Wang, Xin Gui, and Xiangchuan Gao, "Robust downlink beamforming and power splitting design in multiuser MISO SWIPT system," in Proc IEEE ICCC, Oct. 2014, pp. 271-275.

[6] Q. Shi, L. Liu, W. Xu, and R. Zhang, "Joint transmit beamforming and receive power splitting for MISO SWIPT systems," IEEE Trans. Wireless Commun., vol. 13, no. 6, pp. 3269-3280, Jun. 2014.

[7] M.R.A. Khandaker and Kai-Kit Wong, "SWIPT in MISO multicasting systems," IEEE Wireless Commun. Lett., vol. 3, no. 3, pp. 277-280, Jun. 2014.

[8] Qingjiang Shi, Weiqiang $\mathrm{Xu}$, Tsung-Hui Chang, Yongchao Wang, and Enbin Song, "Joint beamforming and power splitting for MISO interference channel with SWIPT: An SOCP relaxation and decentralized algorithm," IEEE Trans. Signal Process., vol. 62, no. 23, pp. 6194-6208, Dec. 2014.

[9] N. D. Sidiropoulos, T. N. Davidson, and Z.-Q. Luo, "Transmit beamforming for physical-layer multicasting," IEEE Trans. Signal Process., vol. 54, no. 6, pp. 2239-2251, Jun. 2006.

[10] A. H. Phan, H. D. Tuan, H. H. Kha, and D. T. Ngo, "Nonsmooth optimization for efficient beamforming in cognitive radio multicast transmission," IEEE Trans. Signal Process., vol. 60, no. 6, pp. 2941-2951, Jun. 2012.

[11] U. Rashid, H. D. Tuan, and H. H. Nguyen, "Relay beamforming designs in multi-user wireless relay networks based on throughput maximin optimization," IEEE Trans. Commun., vol. 51, no. 5, pp. 1734-1749, 2013.
[12] H. Tuy, Convex Analysis and Global Optimization, Kluwer Academic, 2001.

[13] P. Apkarian, D. Noll, and A. Rondepierre, "Mixed $h_{2} / h_{\infty}$ control via nonsmooth optimization," IEEE Trans. Signal Process., vol. 47, no. 3, pp. 1516-1546, Mar. 2008.

[14] A. B.-Tal and A. Nemirovski, Lectures on Modern Convex Optimization, SIAM, 2001.

[15] H. D. Tuan, P. Apkarian, S. Hosoe, and H. Tuy, "D.C. optimization approach to robust control: Feasibility problems," Int. J. Contr, vol. 73, no. 2, pp. 89-104, Feb. 2000.

[16] A. A. Nasir, D. T. Ngo, H. D. Tuan, and S. Durrani, "Iterative optimization for joint transmit beamforming and receive power splitting in small-cell networks with SWIPT," submitted to IEEE Trans. Wireless Commun., 2015.

[17] H. Morosa, H. Harada, A. Morimoto, S. Nasata, H. Ishii, and Y. Okumura, "Cell identification performance based on hierarchical synchronization channels in dense small cell environment," in Proc. IEEE SPAWC, Jun. 2013, pp. 734-738. 